\title{
23
}

\section{Design and Implementation of an ATM Cell Controller for FR/ATM Interworking System}

\author{
Do-Yeon Kim and Jung-Sik Kim \\ Electronics and Telecommunications Research Institute \\ 161 Kajong-Dong, Yusong-Gu,Taejon, 305-350, KOREA \\ Tel : +82 428605349 Fax: +82428606224 \\ E-mail :dykim@nice.etri.re.kr
}

\begin{abstract}
In this paper, we described on the design and implementation of an ATM cell controller for a high speed interface between FRIM (Frame Relay Interworking Module) and ALS (ATM Local switching Subsystem). In upstream direction, the ATM cell controller receives a cell stream from 16 Frame Relay subscriber access boards, and performs UPC (Usage Parameter Control), cell head translation, EHEC generation, AAL type 5 segmentation, and ATM switch interface function. In reverse direction, the ATM cell controller classifies the received cell stream through an ATM switch into user cells and IPC (Inter Processor Communication) cells, and performs 64/53 octets conversion, AAL type 5 cell reassembly, HEC generation, and handling of user cells, IPC cells, and OAM cells. And these functions are implemented by using FPGA, and we have verified the whole ATM cell controller functions by connecting protocol tester, Frame Relay subscriber board and an ATM switch.
\end{abstract}

Broadband Communications P. Kühn \& R. Ulrich (Eds.)

(C) 1998 IFIP. Published by Chapman \& Hall 


\section{Keywords}

ATM, Frame Relay, Interworking, ATM Cell Controller

\section{INTRODUCTION}

Early ATM systems serve as a backbone mainly for data communications. Therefore existing or upcoming data services, such as X.25, Frame Relay, and SMDS (Switched Multi-megabit Data Service) have to be supported. Frame Relay is an enhanced packet-type service. Higher throughput and less delay are achieved by reducing error control and forgoing end-to-end flow control. Frame Relay is a connection-oriented service offering bit rates from some $\mathrm{Kbit} / \mathrm{s}$ up to $2 \mathrm{Mbit} / \mathrm{s}$ or possibly higher.

The merit of an ATM backbone network for a network operator is that a common, unique network infrastructure can be deployed flexibly to support all the existing and future services. New switching systems are thus needed to handle not only new ATM-based services but also existing services, such as FRS (Frame Relay Service), POTS and N-ISDN (Narrowband Integrated Services Digital Network) services. Recently with these trends, the concept of FROA (Frame Relay Over ATM) has been actively discussed.

In Korea, Frame Relay commercial services were started last year. And we are constructing a national high speed network with ATM. But actual services on the ATM network are uncertain except existing Frame Relay. This forces ATM switching system to include Frame Relay interworking function. For interworking implementation, we refer to implementation agreements of Frame Relay Forum and ITU-T recommendation.

The content of this paper is organized as follows. In section 2, we describe the overall system configurations for the Frame Relay service over ATM networks. In section 3, we show the results of estimating the number of connections in FRIM. In section 4, we describe an ATM cell controller structure. In section 5, we describe an ATM cell controller functions. And we finish our paper with conclusions in section 6.

\section{SYSTEM CONFIGURATION}

\subsection{Overall system architecture}

Figure 1 shows the configuration of ATM switching system with FRIM implemented for frame relay service access. The switching system has 3-stages configuration as ALS-ACS-ALS. Here ACS (ATM Central switching Subsystem) plays a central role of whole system such as loading and management and supports to expand system capacity, and ALS (ATM Local switching Subsystem) plays a role of local switch with complete signaling processors and usually accommodates UNI/NNI. The operation terminal (WS) and the OMP (Operation and Maintenance 
Processor) are connected to each other by an Ethernet bus. The operation terminal provides operator with various graphical input/output functions. FRIM is a kind of IWF (Inter Working Function) and it is connected to ALS via IMI (Inter Module Interface) as a part of switching system. FRIM supports all Frame Relay interworking functions except call control signaling. The IMI is common bus between subsystem and functional module and it is used to transfers IPC cells as well as user cells with $155 \mathrm{Mbps}$ rate. The internal cell has 3 bytes routing tag in front of usual ATM cell to indicate the port of ALS/ACS switching system. Our internal cell format has 56 bytes/cell logically but 64 bytes/cell physically. The routing tags are assigned by CCCP (Call Connection Control Processor) of ALS at call setup. The call control signaling including Q.933-to-Q.2933[7] mapping are supported by the CCCP.

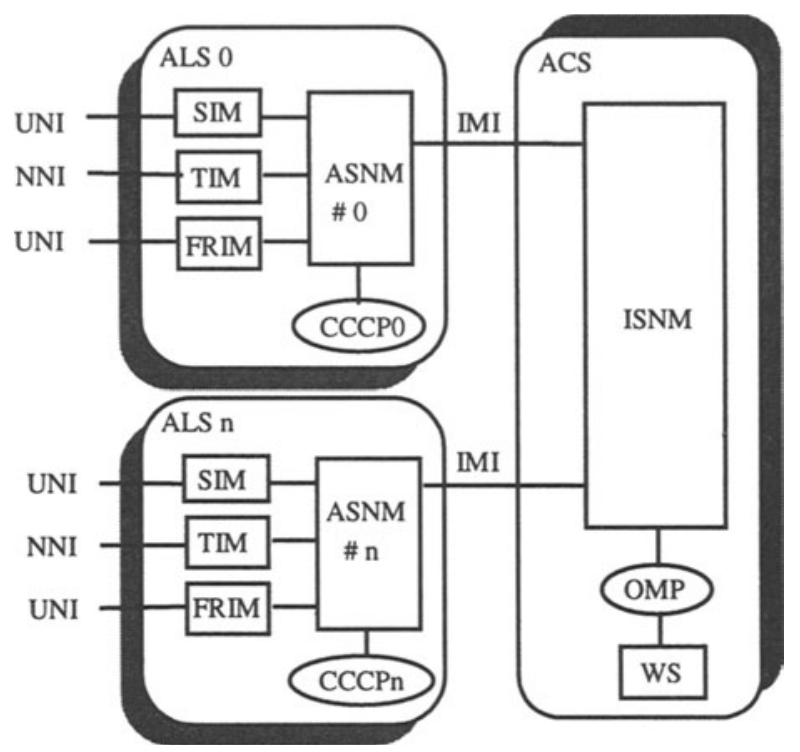

Figure 1 ATM switching system for FR/ATM interworking

\subsection{Frame relay interworking module}

FRIM consists of three kinds of functional boards such as ATM cell control board, system clock distribution board and Frame Relay subscriber access board as shown in Figure 2. ATM cell control board performs cell multiplexing and demultiplexing function, AAL type 5 function for IPC between upper processors, and other ATM common functions such as UPC, VPI/VCI translation and OAM cell detection/generation. Frame Relay subscriber access board performs channelized DS1/E1 interfaces, Q.922 core function, frame to ATM cell conversion function, network layer protocol processing, management translation between OAM and 
PVC status, and Frame Relay/ATM interworking core function. The board is used at UNI and NNI. System clock distribution board performs module clock generation from system clock which is derived from $155.52 \mathrm{MHz}$. Within FRIM, cell bus is implemented by 16 bits width FIFO communication to transfer user cells. IPC bus is implemented by 16 bits width DPRAM to transfer module maintenance information including board status, DS1/E1 alarm status, hardware configuration data, connection setup data and status change of each connection.

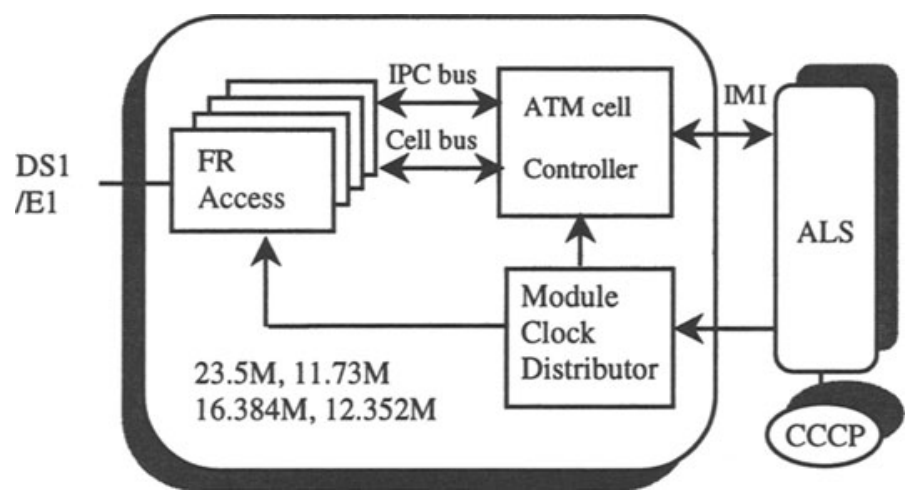

Figure 2 Frame relay interworking module

\section{ESTIMATING NUMBER OF CONNECTIONS}

FRIM has 64 DS1/E1 interfaces. The multiplexed traffic is $128 \mathrm{Mbps}$ that is less than STM1. Each E1 has 31 channels of $64 \mathrm{kbps}$ in channelized Frame Relay. We assign 16kbps to minimum CIR (Committed Information Rate) for PVC. This means FRIM accommodates maximum 7936 connections. But the number may be reduced to some reasonable amount like 4096. This is dependent on how wide the user traffic are. The user traffic trends are getting wide in accordance with diverse multimedia services. On the other hand, 1024 connections are regarded reasonable for STM1 ATM UNI. Supporting more connections in our FRIM is the capacity of ATM cell controller. We need to calculate the number of Frame Relay PVC connections in our system. The number of connections are calculated by the following equation.

Number of Connections $=\frac{\text { Traffic }}{\sum_{i} P(i) \times \operatorname{CIR}(i)}$

where 
Traffic $=64 \times 31 \times 64 \mathrm{kbps}$

$\mathrm{P}(\mathrm{i})=$ probability of CIR(i)

CIR(i) $=$ CIR value for $i$-th item

We obtained PVC distribution among CIR from Frame Relay Forum of Japan. We used it for an initial value. To estimate the distribution of near future, we used the iteration scheme as following equation.

where

$$
\mathrm{P}(\mathrm{n}, \mathrm{m})=(1-\mathrm{w}) \times \mathrm{P}(\mathrm{n}, \mathrm{m}-1)+\mathrm{w} \times \mathrm{P}(\mathrm{n}-1, \mathrm{~m}-1)
$$

$\mathrm{P}(\mathrm{n}, \mathrm{m})=$ Probability of $\operatorname{CIR}(\mathrm{n})$ at $\mathrm{m}$-th iteration

$\mathrm{P}(\mathrm{n}, \mathrm{m}-1)=$ Probability of $\operatorname{CIR}(\mathrm{n})$ at $(\mathrm{m}-1)$-th

iteration

$\mathrm{P}(\mathrm{n}-1, \mathrm{~m}-1)=$ Probability of $\mathrm{CIR}(\mathrm{n}-1)$ at $(\mathrm{m}-1)$-th

iteration

$\mathrm{w}=$ weighting factor

This equation assumes that some connections enlarge their bandwidth to the next CIR at each iteration. Figure 3 shows the estimated number of Frame Relay connections with $w=0.1$ in our FRIM. The CIR ranges from 0 to $1.5 \mathrm{Mbps}$. With the present distribution, over 4800 connections are required. At iteration 6 we gained 4096 connections, and its dominant CIRs range from $32 \mathrm{kbps}$ to $256 \mathrm{kbps}$. The number of connection may become less, if we give constraint to the allowed CIR such as $16 \mathrm{kbps}, 32 \mathrm{kbps}, 64 \mathrm{kbps}, 128 \mathrm{kbps}, 192 \mathrm{kbps}, 256 \mathrm{kbps}, 384 \mathrm{kbps}$, $512 \mathrm{kbps}, 768 \mathrm{kbps}, 1 \mathrm{Mbps}$ and $1.5 \mathrm{Mbps}$.

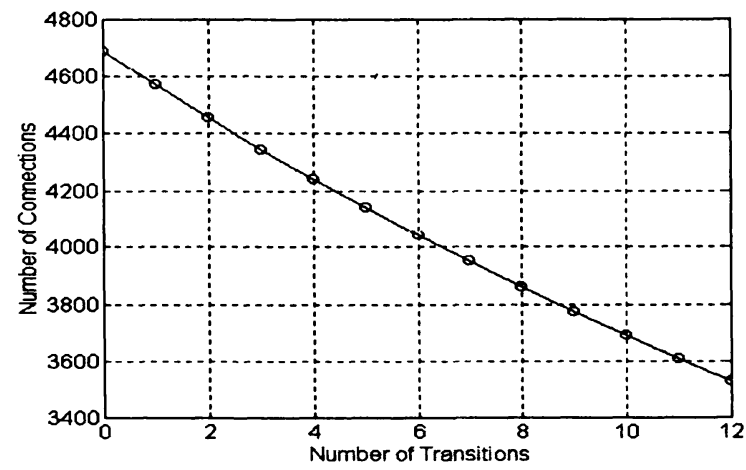

Figure 3 Frame relay connections for $64 \mathrm{E} 1(\mathrm{w}=0.1)$

\section{THE STRUCTURE OF ATM CELL CONTROLLER}

The structure of an ATM cell controller is shown in Figure 4. It is composed of a CBRI (Cell Bus Receive Interface), two CAMs (Content Addressable Memory), a 
UPC processor, a DPRAM, HCTI (Head translation and Cell Transmission IC), an AAL type 5 Segmentation/Reassembly Device, 256 Kbyte Packet/Control Memory, 512 Kbyte program memory, 4 Mbyte data memory, a MPU, a BAAI (Bus Arbitration and Address decoding IC), a LTRI (Link Transmitter/Receiver IC), a Clock Receiver, an ALI (ATM Link Interface IC), an IPC cell receive FIFO, and others.

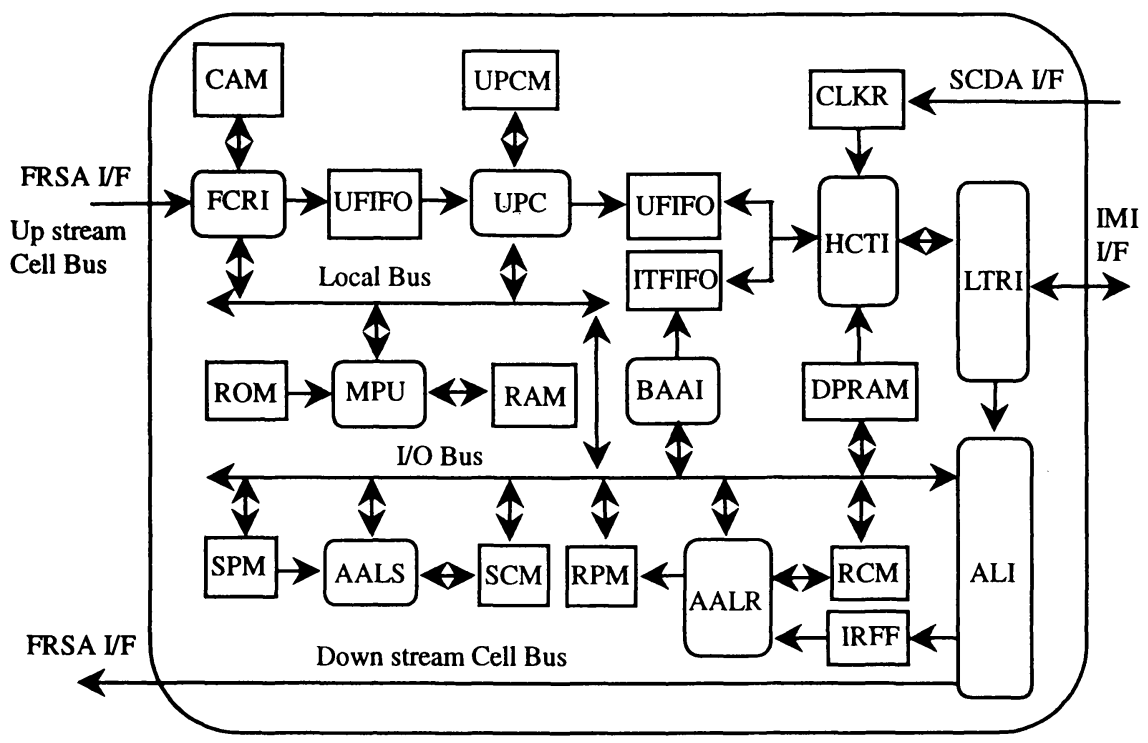

Figure 4 The structure of ATM cell controller

The ATM cell controller has 4 interfaces. They are a cell bus interface, an IPC bus interface, an IMI (Inter Module Interface), and a clock interface. The CBRI reads data from synchronous FIFO in Frame Relay subscriber access board when the CBRI detects cell available flag. In this case, cell boundary is classified by start of cell signal. Incoming cell stream has 20 variable bits in cell head, which are BD3BD0, LI1-LI0, SB4-SB0, DL9-DL0, PTI, CLP, HEC, and cell payload. The incoming cell format is shown in Figure 5.

We need to translate incoming cell to connection identifier. Because of incoming cell stream has too many variable bit, the number of connection is dependent on how wide the user traffic are. In section 3, we estimated the number of connections. By the result, an ATM cell controller supports maximum 4,096 connections. For this we used two $2 \mathrm{~K}$ x 64 bits CAMs (Content-addressable Memory). The CAM, also known as Associative Memory, operate in the converse way to Random Access Memories. In a RAM, the input to the device is an address, and the output is the data stored at that address. In a CAM, the input is a data sample and the output is a flag to indicate a match and the address of the matching data. 


\begin{tabular}{|c|c|c|c|c|c|c|c|c|}
\hline & 7 & 6 & 5 & 4 & 3 & 2 & 1 & 0 \\
\hline 1 & $\mathrm{X}$ & $X$ & $\mathrm{X}$ & $\mathrm{X}$ & $\mathrm{X}$ & $\mathrm{X}$ & BD3 & BD2 \\
\hline 2 & BD1 & BD0 & LI1 & $\mathrm{LIO}$ & $\mathrm{X}$ & SB4 & SB3 & SB2 \\
\hline 3 & SB1 & SB0 & DL9 & DL8 & $\overline{\text { DL7 }}$ & DL6 & DL5 & $\overline{\text { DLA }}$ \\
\hline 4 & DL3 & DL2 & DL1 & DLO & & PTI & & CLP \\
\hline 5 & \multicolumn{8}{|c|}{ HEC } \\
\hline & \multicolumn{8}{|c|}{ Payload 0} \\
\hline & \multicolumn{8}{|c|}{ Payload n } \\
\hline 53 & \multicolumn{8}{|c|}{ Payload 47} \\
\hline
\end{tabular}

BD3-BD0 : Board Identifier(0 to 15$)$

LI1-LI0 : Link Identifier(0 to 3 )

SB4-SB0 : Subscriber Identifier(0 to 30$)$

DL9-DL0 : DLCI(0 to 1023)

$X$ : Not used

Figure 5 Incoming cell format

We use CAM mode because the CAM mode allows large address space to be searched rapidly and efficiently. Also, the CBRI provides CAM control signal and it writes ATM cell into UPC FIFO. In this case, the VPI/VCI of cell is connection identifier generated by the CAM. Figure 6 shows UPC input and output cell format.

\begin{tabular}{|c|c|c|c|c|c|c|c|c|}
\hline & 7 & 6 & 5 & 4 & 3 & 2 & 1 & 0 \\
\hline 1 & \multicolumn{8}{|c|}{ Not used } \\
\hline 2 & \multicolumn{8}{|c|}{ Not used } \\
\hline 3 & CI11 & CI10 & $\mathrm{Cl} 9$ & $\mathrm{CI} 8$ & $\mathrm{CI} 7$ & CI6 & CI5 & CI4 \\
\hline 4 & $\mathrm{Cl} 3$ & $\mathrm{Cl} 2$ & CI1 & $\mathrm{ClO}$ & & PTI & & CLP \\
\hline 5 & \multicolumn{8}{|c|}{ HEC } \\
\hline & \multicolumn{8}{|c|}{ Payload 0} \\
\hline & \multicolumn{8}{|c|}{ Payload n } \\
\hline 53 & \multicolumn{8}{|c|}{ Payload 47} \\
\hline
\end{tabular}

CI11-0 : Connection identifier (0 to 4095)

Figure 6 UPC in/out cell format

UPC processor checks incoming connections for violations of negotiated traffic parameters, and selectively discards cells in violation or tags cells in violation with Cell Loss Priority (CLP) $=1$ on a per connection basis. The UPC processor transfer cells through standard UTOPIA FIFO interface and maintains the following counts 
per connection : total cells, CLP $=0$ cells, and cells in violation. The UPC processor supplies two GCRA (Generic Cell Rate Algorithms) engines. The GCRA is a continuous leaky bucket algorithm in which cells leak from the bucket at a continuous rate. The bucket state reflects the amount of time required for the bucket to empty. The bucket size is the maximum amount of time allowed for the bucket to empty. If cells arrive faster than they leak out, the level of the bucket rises. Once the bucket becomes full, arriving cells are determined to be in violation. If cells arrive slower than they leak out, the level of the bucket sinks toward empty. The transferred user data is saved into UFIFO (User FIFO). When 53 octets user data is saved, almost full flag of UFIFO is activated to low. IPC FIFO saves OAM cell and IPC cell. Both OAM cell and IPC cell are segmented by AAL type 5 segmentation device. The AAL5 segmentation device performs the functions necessary to segment frames into ATM cells.

HCTI saves a cell to temporary register when an almost FIFO full flag of either user FIFO or IPC FIFO is detected. If the almost UFIFO full flag is received, the HCTI reads a cell with 8 bit data format from UFIFO and makes address using VPI/VCI in cell. The address is pointer to read head translation table in DPRAM. The head translation table has 3 bytes routing tag and 4 bytes outgoing VPI/VCI as shown Figure 7. After head translation, the HCTI sends reformatted cell to the LTRI (Link Transmitter and Receiver IC). If the almost FIFO full flag is not received, the HCTI sends IDLE pattern to the LTRI. The HCTI also provides EHEC (Extended HEC) code, odd parity bit to the LTRI. The HCTI performs EHEC calculation over 7 octets ( 3 bytes routing tag +4 bytes cell header) of internal cell header for the verification of the header information. The EHEC is inserted into the HEC field of the cell header. The polynomial for EHEC is $\mathrm{X}^{8}+\mathrm{X}^{2}+\mathrm{X}+1$.

\begin{tabular}{|c|c|c|c|}
\hline Connection & 15 & 8 & 7 \\
\hline$\times 4$ & \multicolumn{2}{|c|}{ Not used } & Routing Information 0 \\
\hline 1 & \multicolumn{2}{|c|}{ Routing Information 1} & Routing Information 2 \\
\hline 2 & Not used & \multicolumn{2}{|c|}{ Outgoing VPI } \\
\hline 3 & \multicolumn{3}{|c|}{ Outgoing VCI } \\
\hline $\mathrm{n}$ & \multicolumn{2}{|c|}{ Not used } & Routing Information 0 \\
\hline
\end{tabular}

Figure 7 The translation table of cell header

LTRI performs IMI (Inter Module Interface) functions. The IMI has functions such as digital phase alignment, elastic buffer, cell synchronization, encode/decode, parallel to serial conversion and serial to parallel conversion, link redundancy control, and maintenance and administration to obtain highly reliable interface. It also checks both EHEC (Extended HEC) bit and parity bit, and then reports the 
results of the check to the HCTI. Clock receiver receives $46.9494 \mathrm{MHz}$ and $11.7374 \mathrm{MHz}$ clock which is generated by the system clock distribution board and provides it to LTRI. Using divider, the clock receiver also provides $23.4747 \mathrm{MHz}$ clock to the CBRI, HCTI etc. At this time the $23.4747 \mathrm{MHz}$ clock is given by $155.52 \mathrm{MHz} \times$ 64/53octets. MPU registers the incoming VPI/VCI into CAM to search connection identifier and it loads 3 bytes routing tag and 4 bytes outgoing VPI/VCI in DPRAM. BAAI performs data bus arbitration between processor and AAL type 5 device and address decoding function.

ALI receives a cell from LTRI. The received cell format is shown in Figure 8. When a cell is received, ALI checks MSB of the first octet. If the MSB is 0, ALI classifies either a user cell or a IPC cell checking third octet. If incoming cell is user cell, ALI checks PTI field of cell header. If the PTI is b"100" (segment OAM) or b" 101 " (end to end OAM), incoming cell is written into IPC receive FIFO. If the PTI is neither b"100" nor b" 101 ", incoming cell is transferred to cell bus. The cell bus is connected to Frame Relay subscriber access board. Also, IPC cell is written into IPC receive FIFO. And ALI performs HEC calculation over 4 octets of incoming cell header for the verification of the header information. The AAL5 reassembly device performs the functions necessary to make ATM cells into segment frames.

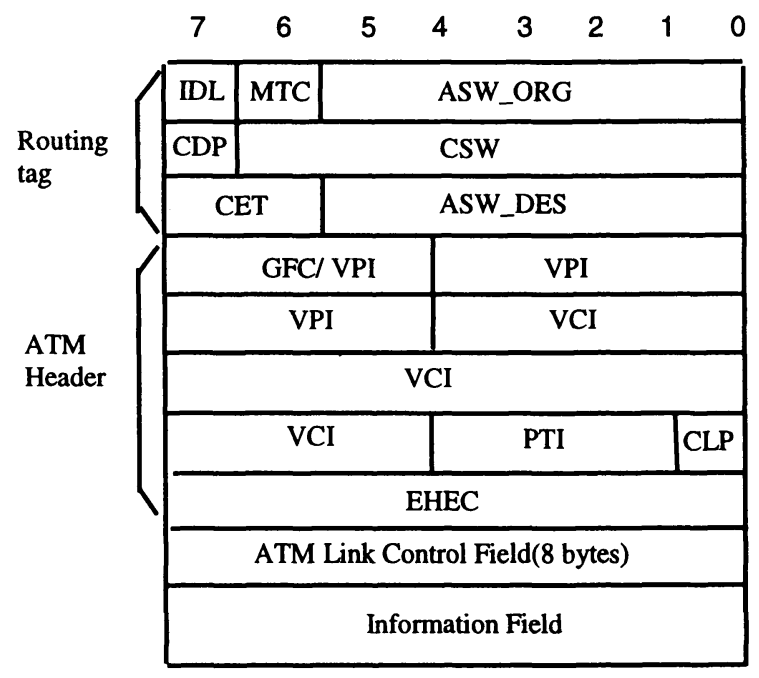

IDL : Idle cell ID(0:Busy, $1:$ Idle)

MTC : Multicast cell(1:p-t-p, 0:p-t-mp)

CDP : Cell Delay Priority ID

CET : Cell type ID(00:User information cell, 01, 10: Controller cell, 11: IPC cell)

ASW_ORG : Access Switch Origin address

CSW : Central Switch physical address

ASW_DES : Access Switch Destination address

MCN : Multicasting Channel Number

Figure 8 Internal cell format 
The CBRI, HCTI, BAAI, and ALI was implemented by using a FPGA. It was described using HDL code and their logic gates were generated by a synthesis tool. They were simulated by a logic simulation tool.

5 The fuctions of ATM cell controller

An ATM cell controller contains the following functions.

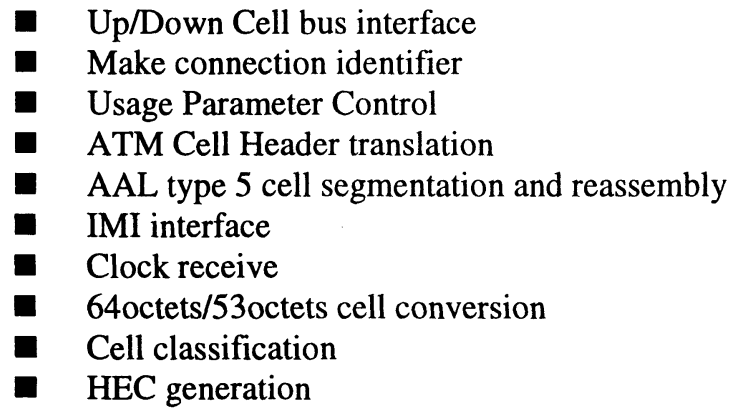

\section{CONCLUSION}

We have implemented an ATM cell controller to provide a high speed interface between FRIM and ALS. The ATM cell controller performs cell multiplexing and de-multiplexing functions, AAL type 5 function for IPC between upper processors, and other ATM common functions such as UPC, VPI/VCI head translation and OAM cell detection/generation. The ATM cell controller is composed of FPGA, CAM, UPC processor, DPRAM, FIFO, AAL type 5 segmentation and reassembly device, program/data memory, MPU, LTRI, ALI, BAAI, and others. We have verified the whole ATM cell controller functions by connecting a protocol tester, Frame Relay subscriber board and an ATM switch. Also, this ATM cell controller may be applicable to the development of VTOA.

\section{REFERENCES}

Rainer Handel, Manfred N. Huber, and Stefan Schroder (1994) ATM Networks: Concept, Protocols, Applications, Addison-Wesley Publishing Company, Wokingham.

ITU-T Rec. I.555 (1995) Frame Relaying Bearer Service Interworking.

David Ginsburg (1996) ATM, Solutions for enterprise internetworking, in Addison Wesley, pp.269-275.

Frame Relay Forum (1994) Frame Relay/ATM PVC Network Interworking Implementation Agreement, Frame Relay Document FRF.5. 
Frame Relay Forum (1994) Frame Relay/ATM PVC Service Interworking Implementation Agreement, Frame Relay Document FRF.8.

Hong-Shik Park, Yool Kwon, Young-Sup Kim, and Seok-Youl Kang (1997) ATM Interface Technologies for an ATM Switching System, ETRI Journal, Vol. 18, No. 4.

ITU-T Rec. Q.2933 Draft (1991) BISDN-DSS2-Signalling Specification for Frame Relay Service. 